\title{
Stabilization of all-trans-lycopene from tomato by encapsulation using cyclodextrins
}

\author{
Gracia Patricia Blanch ${ }^{\mathrm{a}, *}$, María Luisa Ruiz del Castillo ${ }^{\text {a }}$, María del Mar Caja ${ }^{\mathrm{a}}$, \\ Mercedes Pérez-Méndez ${ }^{b}$, Santiago Sánchez-Cortés ${ }^{c}$ \\ ${ }^{a}$ Instituto de Fermentaciones Industriales, CSIC, c/Juan de la Cierva, 3, 28006 Madrid, Spain \\ ${ }^{\mathrm{b}}$ Instituto de Ciencia y Tecnología de Polímeros, CSIC, c/Juan de la Cierva, 3, 28006 Madrid, Spain \\ ${ }^{\mathrm{c}}$ Instituto de Estructura de la Materia, CSIC, c/Serrano 121, 28006 Madrid, Spain
}

Received 23 November 2006; received in revised form 10 April 2007; accepted 29 April 2007

\begin{abstract}
The stabilization of all-trans-lycopene from tomato by encapsulation using $\alpha$-, $\beta$ - and $\gamma$-cyclodextrins (CDs) was evaluated. To that end, two different encapsulation methods were comparatively studied: a conventional method and a supercritical fluid extraction (SFE) process. An optimization procedure considering distinct molar ratios of CD/lycopene (1/0.0026, 1/0.005 and 1/0.05) as well as the type of cyclodextrin to be used was accomplished. The encapsulation was determined by using micro-Raman spectroscopy. All-trans-lycopene employed was obtained by SFE with a purity around $90-95 \%$. As a result, a molar ratio CD/lycopene of $1 / 0.0026$ was selected as it provided the best complexation yields $(93.8 \%)$ whilst $\beta$-CD seemed to be the most favorable to be used to stabilize lycopene. A comparison of the two methods studied reflected higher encapsulation yields from the conventional method. However, the supercritical fluid approach offers numerous advantages such as the possibility of performing the extraction, fractionation and encapsulation of lycopene from tomato in one step, shortening notably the overall procedure time and minimizing the sample handling.
\end{abstract}

(c) 2007 Elsevier Ltd. All rights reserved.

Keywords: Encapsulation; All-trans-lycopene; Cyclodextrin; Supercritical fluid extraction

\section{Introduction}

The biological and nutritional importance of food compounds has been the subject of intense research during the last years. Carotenoid supplementation offers great interest because of their characteristics as antioxidant agents since they can prevent heart diseases and regulate the immuno system. Specifically, lycopene has been demonstrated to have stronger antioxidant capacity than that $\beta$-carotene due to its great ability to trap peroxyl radicals (Di Mascio, Katser, \& Sies, 1989; Stahl \& Sies, 1996). However, it has also been studied that the bio-availability of all-trans-lycopene from foods is lower than that of cis-lycopene (Shi \& Le Maguer, 2000) and that "in-vivo" mechanism promotes

\footnotetext{
${ }^{*}$ Corresponding author. Tel.: +34 9156229 00; fax: +34 915644853 . E-mail address: gblanch@ifi.csic.es (G.P. Blanch).
}

isomerization of lycopene from trans to cis form (Nguyen \& Schwartz, 1999). Besides, all-trans-configuration, which is thermodynamically more stable, appears to clearly predominate in nature (Chandler \& Schwartz, 1987; Wilberg \& Rodriguez Amaya, 1995). All in all, lycopene extraction techniques and storage conditions that control its degree of isomerization are at present required.

In this context, encapsulation is used to protect compounds from oxidation, evaporation and off-flavor. The encapsulated compounds can be presented either with spherical geometry (single-particle structure) or with irregular geometry (aggregated structure) (Pedrolas Islas, 2002; Shahida \& Han, 1993). The most used mechanisms in encapsulation are extrusion, coacervation and complexation with e.g. cyclodextrins (CDs). The application of CDs for molecular encapsulation in foods offers several advantages as they possess macrocycles which present a 
torus-shaped structure with an adaptable hydrophobic cavity into which lipophilic guest molecules can be hosted (Mcmullan, Saenger, Fayos, \& Mootz, 1973). Besides, they are non-toxic, inexpensive, thermally stable until $100^{\circ} \mathrm{C}$ and not hygroscopic. Additional advantages are the fact that they are not absorbed in the upper gastrointestinal tract and that are completely metabolized by the colon microflora (Szente \& Szejtli, 2004). In fact, $\beta$-cyclodextrin has been recently approved for food use in several countries, which has resulted obviously in a growing interest of its use in food-related applications. In this respect, the capability of cyclodextrins to form inclusion complexes with a wide variety of guest molecules (Garbow, Likos, \& Schroeder, 2001; Li \& Purdy, 1992; Rekharsky \& Inove, 1998) and, specifically, with carotenoids (Basu \& Del Vecchio, 2001) is known. In particular, associations of CDs with $\beta$-carotene or lycopene have already been published by some authors (Mele, Mendichi, \& Selva, 1998, 2002). However, reports on the encapsulation with CDs by supercritical fluids, to our knowledge, are scarce in the literature. Actually, the main application has been recently described by other authors (Perrut, Jung, \& Leboeuf, 2005), who have demonstrated the advantages of the simultaneous use of CDs and supercritical fluids for the enhancement of the dissolution of poorly soluble compounds other than carotenoids.

On the other hand, Resonance Raman Spectroscopy is an interesting technique for the study of carotenoids, in general, and lycopene in particular (Withnall, Chowdhry, Silver, Edwards, \& de Oliveira, 2003), because of its sensitivity to this type of compounds. We report here the application of Raman Resonance technique to the study of lycopene encapsulation with cyclodextrins. In this sense, Raman spectroscopy can provide valuable and specific molecular information about the interaction mechanism taking place in molecular recognition problems (Leyton et al., 2004).

One advantage of Raman spectroscopy in relation to the structural study of lycopene and the CD complex is the fact that the laser line selected to register the Raman spectra $(785 \mathrm{~nm})$ is far enough from the main lycopene absorbance band. Consequently, this may allow to obtain intense preResonance Raman spectra from lycopene without overlapping of the CD Raman bands.

The objective of this work was to perform for the first time the selective extraction, fractionation and encapsulation into CD of all-trans-lycopene from tomato by supercritical fluid extraction (SFE) in one step. A further purpose was to study the stability and isomerization of the complex by micro-Raman spectroscopy.

\section{Materials and methods}

\subsection{Materials}

Tomatoes (Romana variety) were obtained from the local market and maintained at low temperature $\left(6-8^{\circ} \mathrm{C}\right)$ until their use. Subsequently, the skin was separated from the rest of the sample, frozen $\left(-30^{\circ} \mathrm{C}\right)$ and dried in a freeze-dryer (Virtis FM-12XL). The dry residue was ground and stored with nitrogen at $-30{ }^{\circ} \mathrm{C}$ until the extraction was performed. $\alpha$-, $\beta$ - and $\gamma$-cyclodextrins, used without any further purification, were purchased from Fluka (Spain) and all-trans-lycopene (95\% purity) from Sigma Chemical (Spain). Carbon dioxide $\left(\mathrm{CO}_{2}>99 \%\right.$ purity), used as the supercritical fluid, was obtained from Carburos Metálicos (Madrid, Spain).

\subsection{Supercritical fluid extraction of lycopene}

Extractions were performed using a SFE module designed by Iberfluid (Madrid, Spain). It consists of a pump (450 bar), a flow meter, a $300 \mathrm{ml}$ extraction vessel and two $100 \mathrm{ml}$ separation vessels. The temperature and pressure were controlled by metering valves. The extraction system was fully automated and controlled by the ADKIR program. Our previous experience was used to select the extraction procedure (Gómez-Prieto, Ruiz del Castillo, Santa-María, Blanch, \& Herraiz, 2002). Specifically, $50 \mathrm{~g}$ of dry tomato skin was loaded into the extraction vessel while a $\mathrm{CO}_{2}$ stream flowed through the sample at $50{ }^{\circ} \mathrm{C}$ and 320 bar. The first separation vessel was maintained at $40^{\circ} \mathrm{C}$ and 150 bar whereas the temperature and pressure corresponding to the second separation vessel was $25^{\circ} \mathrm{C}$ and $60 \mathrm{bar}$, respectively. The extracts from both separation vessels were dissolved in hexane, the overall extraction time being $3 \mathrm{~h}$. The total contents of both lycopene and other carotenoids used as a reference were estimated from the extraction procedure described elsewhere (AOAC, 1984) and high performance liquid chromatography (HPLC) analysis of the extracts as detailed below.

\subsection{HPLC analysis of SF-extracts}

Before its analysis by HPLC, the extracts obtained by SFE were firstly evaporated under a nitrogen stream, then re-dissolved in $10 \mathrm{ml}$ of a mixture of $0.5 \mathrm{ml}$ of dichloromethane (Labscan) and methanol:methyl-tertbutyl ether (3:1) and finally filtered through a $0.45 \mu \mathrm{m}$ Pro-X filter (Teknokroma, Spain). The HPLC analyses were performed using a Beckman (Fullerton CA, model 126) equipment fit with a $250 \mathrm{~mm} \times 4.6 \mathrm{~mm}$ Develosil UG $\mathrm{C}_{30}$ column (Nomura Chemical, Japan), a manual injection valve (Rheodyne, model 125, Cotati, CA) and a photodiode array detector (Beckman 168). As the mobile phase, a mixture of eluent A (methanol-water, 96:4, v/v) and eluent B (methyl-tert-butyl ether) was used. A linear gradient was applied in $60 \mathrm{~min}$, from $\mathrm{A}: \mathrm{B}(83: 17, \mathrm{v} / \mathrm{v})$ to $\mathrm{A}: \mathrm{B}(33: 67, \mathrm{v}: \mathrm{v})$ using $1.0 \mathrm{ml} / \mathrm{min}$ and $20 \mu \mathrm{l}$ as the flow rate and sample injection volume, respectively. The samples were monitored at 285, 347, 450 and $472 \mathrm{~nm}$ using $\beta$-apocarotenal as an internal standard. Carotenoid identification was made by matching the UV/vis absorption spectra with the respective stan- 
dard compounds while the quantification was carried out using a calibration line obtained from all-trans-lycopene standard.

\subsection{Encapsulation of all-trans-lycopene extracted by SFE}

\subsubsection{Conventional procedure}

The conventional encapsulation procedure was based on that described by other authors (Basu \& Del Vecchio, 2001) and was carried out using $\alpha-, \beta-$ and $\gamma-\mathrm{CD}$. Specifically, each cyclodextrin was dissolved in de-ionized water at $40-45^{\circ} \mathrm{C}$ with agitation. The solution was cooled and the SF-extract of lycopene diluted in $1 \mathrm{ml}$ of ethyl acetate was added. The reaction was carried out under nitrogen atmosphere, at $25^{\circ} \mathrm{C}$ during $16 \mathrm{~h}$ and using stirring. After that, most of the water was removed by liofilization and the resulting precipitate was washed with ethyl acetate to eliminate all the non-encapsulated extract. Finally, the washed precipitate containing the encapsulated $\mathrm{CD}$ with lycopene plus the remaining pure $\mathrm{CD}$ was removed by centrifugation ( $7600 \mathrm{rpm}, 5 \mathrm{~min}$ ), dried under high vacuum and studied by micro-Raman spectroscopy as later detailed. The encapsulation yield (\%) was estimated from the comparison of the initial weight of both CD and all-trans-lycopene used and the amount of non-encapsulated all-trans-lycopene studied by HPLC.

\subsubsection{Supercritical fluid- $\mathrm{CO}_{2}\left(\mathrm{SF}-\mathrm{CO}_{2}\right)$ procedure}

In order to study the encapsulation capacity of $\alpha-\beta$ and $\gamma$-cyclodextrins in SF- $\mathrm{CO}_{2}$, first of all the influence of the $\mathrm{CO}_{2}$ pressure on the pure $\mathrm{CDs}$ was evaluated. For that purpose, a Hewlett-Packard 7680A extraction module was used. A $7.0 \mathrm{ml}$ thick-walled stainless steel thimble, with two porous frits contained in the caps at each end of the thimble allowing to hold the cyclodextrin in place, was employed. A nozzle/trap/assembly (variable restrictor) to allow the pressurization and de-pressurization of the supercritical fluid was used. A 40-mg weight of each CD studied was placed into the extraction vessel at different pressures (i.e., 101, 151 and 245 bar) and distinct temperatures (i.e., 40 and $50^{\circ} \mathrm{C}$ ) during $30 \mathrm{~min}$. Additionally, pressure of 1 bar at room temperature was also studied.

Subsequently, X-ray diffraction in crystalline power of the pure $\alpha$-, $\beta$ - and $\gamma$-cyclodextrins was performed using a powder Philips diffractometer, $25 \mathrm{~mA} 40 \mathrm{kV}$, employing copper radiation at room temperature $\left(\lambda_{\mathrm{Cu}}=1.5418 \AA\right)$. Sweeping speed used was $1^{\circ} / \mathrm{min}$ with slits $1^{\circ} \times 0.2^{\circ} \times 1^{\circ}$.

Once confirmed the structural preservation of $\mathrm{CD}$ with the pressure effect, the all-trans-lycopene encapsulation with $\mathrm{CD}$ using $\mathrm{SF}-\mathrm{CO}_{2}$ was performed by using the SFE module designed by Iberfluid previously described. Similarly, $50 \mathrm{~g}$ of dried tomato skin was loaded into the extraction vessel whereas $400 \mathrm{mg}$ of cyclodextrin was placed into the two separation vessels with the aim of assuring the encapsulation. During this time a $\mathrm{CO}_{2}$ stream passed through the system at $50^{\circ} \mathrm{C}$ and 320 bar. The procedure time including the extraction and the encapsulation by
SFE was $3 \mathrm{~h}$. Finally, the formed complexes were collected from the vessels and subsequently washed by using ethyl acetate. Then, they were centrifuged $(7600 \mathrm{rpm}, 5 \mathrm{~min})$, and washed once more with ethyl acetate to remove all the non-encapsulated lycopene. Finally, the resulting complex was dried under high vacuum and studied as below described. The encapsulation yield (\%) was estimated from the comparison of lycopene amount estimated in the initial dried tomato skin loaded into the extraction vessel and lycopene amount remaining in the sample after extraction. Both amounts of lycopene were calculated according to the extraction procedure detailed in AOAC (1984) followed by the HPLC analysis as described before.

\subsection{Stability of the complexes}

Once formed the encapsulated complexes from the experimental procedures specified above, their stability was evaluated. Specifically, the $\beta$-cyclodextrin/lycopene complex obtained by the conventional procedure was used to this aim. For that purpose, the encapsulated was stored at room temperature, with light and oxygen and analyzed by micro-Raman spectroscopy, as below described, immediately finished the encapsulation and after $24 \mathrm{~h}, 1$ month and 6 months.

\subsection{Complex determination by micro-Raman spectroscopy}

Raman spectra, corresponding to the complex obtained by both the conventional and SF- $\mathrm{CO}_{2}$ procedures, were obtained from a Renishaw Micro-Raman RM2000 spectrometer equipped with a Leica microscope and a CCD camera using the $785 \mathrm{~nm}$ line as excitation line. This excitation wavelength was selected in order to get information from both lycopene and cyclodextrin since at this wavelength a much lower resonance Raman effect arises from the dye, although a pre-resonance Raman is expected. The spectra shown here were obtained by using a NPLAN $100 \times$ objective. A laser power of $2 \mathrm{~mW}$ at the sample was employed. Integration time was $30 \mathrm{~s}$ for each spectrum. The analyzed area with micro-Raman is ca. $2 \mu \mathrm{m}^{2}$.

\section{Results and discussion}

As a result of applying the dry procedure to the tomato skin, a dry sample representing 3\% of the fresh skin was obtained. From SF-extraction of this sample, about 30 $50 \%$ of lycopene with respect to the total lycopene content in the original dry sample was obtained in the first separation vessel, with a purity of $90-95 \%$, whereas $100 \%$ of the rest of carotenoids with respect to the total content of carotenoids in the dry sample was collected in the second separation vessel. From the HPLC analyses of both extracts, it was observed that $90 \%$ of lycopene extracted and collected in the first separation vessel corresponded to all-trans-lycopene. Also, it was obtained as crystals, $10 \mu \mathrm{m}$ being the size observed by electronic microscopy. 
Likewise, the rest of carotenoids in the second separation vessel seemed equally to exhibit trans-configuration.

To perform the complexations of the extracted all-translycopene with $\mathrm{CD}$, different molar ratio $\mathrm{CD}$ :lycopene (1/ $0.0026,1 / 0.005$ and $1 / 0.05$ ) were tested by using $\beta-C D$, the all-trans-lycopene obtained by SFE and the conventional encapsulation procedure previously described in 2 . It was observed that the lower molar ratio $(1 / 0.0026)$ provided the best encapsulation yield $(93.8 \%)$. In fact, the molar ratio of $1 / 0.005$ and $1 / 0.05$ led to yields as low as $31.8 \%$ and $2.4 \%$, respectively. Possibly in this latter case reaction times longer than $16 \mathrm{~h}$ might compensate the higher amount of lycopene used for the higher molar ratio. In any case, this option would result in extremely long reaction times and, therefore, a 1/0.0026 molar ratio was selected to accomplish the encapsulations. This result is in accordance with that published by Matioli and Rodriguez-Amaya (2003), which describe the employment of lycopene of purity similar to that used in the present work.

The conventional procedure of encapsulation, as specified in Materials and Methods, to select the type of cyclodextrin to be used in the preparation of the complexes, was once more followed by using the optimized molar ratio (1/ $0.0026)$ between all-trans-lycopene and $\alpha-, \beta-$ and $\gamma-C D$, respectively. The selection was based on the results obtained by micro-Raman due to the usefulness of Raman spectroscopy in structural studies of carotenoids. Fig. 1 shows the micro-Raman spectra of the complexes obtained from a 1/0.0026 molar ratio of all-trans-lycopene with $\gamma$-, $\alpha-$ and $\beta-C D$ (Fig. 1c-e, respectively) by conventional encapsulation. Besides, the Raman spectra corresponding to pure $\beta-\mathrm{CD}$ and all-trans-lycopene are also included

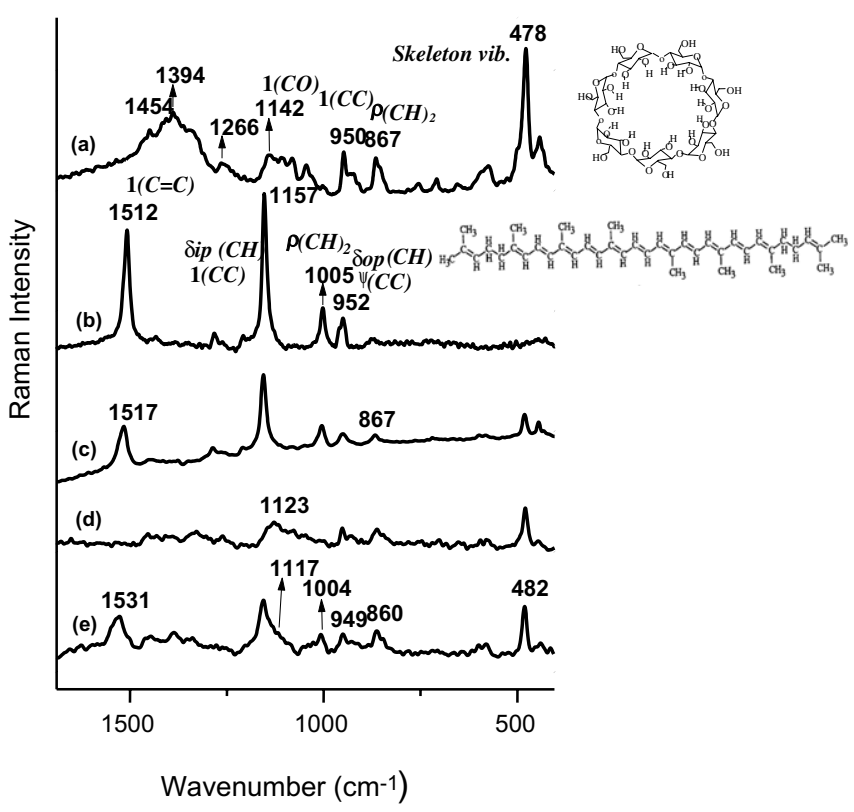

Fig. 1. Micro-Raman spectra representing the band intensity obtained at different wave numbers $\left(\mathrm{cm}^{-1}\right)$ corresponding to $\beta$-cyclodextrin (a), alltrans-lycopene (b) and all-trans-lycopene complex obtained by the conventional method with $\gamma$ - (c), $\alpha$ - (d) and $\beta$-cyclodextrin (e).
(Fig. 1a and b, respectively). It is important to mention that considering that, despite their different size, $\alpha-, \beta-$, and $\gamma$-CD possess similar structure and therefore show a comparable Raman spectrum. As a consequence, $\beta-\mathrm{CD}$ was used as an example to carry out the study on the interaction with lycopene. The results below commented were mainly based on the comparison of the spectra obtained for the encapsulated complexes with that of the pure alltrans-lycopene. Comparisons with $\beta$-CD were only occasionally used because of both its weaker Raman signal and of the presence of pure $\beta-C D$ in the complex as a consequence of its excess in the encapsulation procedure.

As can be seen in Fig. 1a, the characteristic bands of $\beta$ $\mathrm{CD}$ were obtained in the following regions (Tu, 1982): at $1454 \mathrm{~cm}^{-1}$ assigned to $\delta\left(\mathrm{CH}_{2}\right)$ motions; a broad signal between 1400 and $1300 \mathrm{~cm}^{-1}$ attributed to $\delta(\mathrm{OH})$; the bands falling in the $1150-1000 \mathrm{~cm}^{-1}$ region, attributed to $v(\mathrm{CO})$; the bands of the band at $950 \mathrm{~cm}^{-1}$ due to $v(\mathrm{CC})$ motions; and the bands falling in the $850-700 \mathrm{~cm}^{-1}$ due to $\rho\left(\mathrm{CH}_{2}\right)$ and to $\delta(\mathrm{OH})$. The bands below these regions are attributed to molecular skeletal vibrations. It is important to point out that the most intense $\beta-C D$ band appears at $478 \mathrm{~cm}^{-1}$.

Similarly, the bands of all-trans-lycopene are shown in Fig. 1b. Specifically, two intense signals at 1512 and $1157 \mathrm{~cm}^{-1}$, corresponding to the $v(\mathrm{C}=\mathrm{C})$ and in plane $\mathrm{C}-\mathrm{H}$ bending coupled to $v(\mathrm{C}=\mathrm{C})$ and $v(\mathrm{C}-\mathrm{C})$ vibrations (vibrations $(\delta \mathrm{ip}(\mathrm{CH}) / v(\mathrm{CC})$ ) typical of conjugated polyenes (Koyama, 1995), were observed. Other weaker bands were also found at 1005 and $952 \mathrm{~cm}^{-1}$ and are due to $\mathrm{CH}_{3}$ rocking vibrations and out-of-plane $\mathrm{C}-\mathrm{H}$ bending coupled to $v(\mathrm{C}=\mathrm{C})$ and $v(\mathrm{C}-\mathrm{C})$ vibrations vibrations $(\delta \mathrm{op}(\mathrm{CH}) /$ $v(\mathrm{CC})$ ).

Regarding the Raman spectrum of $\alpha$-CD/lycopene complex (Fig. 1d), it is worth mentioning that no signals corresponding to lycopene, between 1150 and $1550 \mathrm{~cm}^{-1}$ were encountered. As a matter of fact, the Raman spectrum is dominated by $\alpha-C D$ bands appearing at $1123 \mathrm{~cm}^{-1}$ and $478 \mathrm{~cm}^{-1}$. Therefore, it might be concluded that it is quite likely that the complex between lycopene and $\alpha$-CD was not formed. Actually, this fact was not surprising since the van der Waals (VDW) section of lycopene is approximately $5.8 \AA$ whereas the VDW hole of $\alpha$-CD is only $5 \AA$ resulting in the disability of the encapsulation of lycopene with $\alpha$-CD.

However, when the lycopene complex was carried out using the other two cyclodextrins employed ( $\beta$ - and $\gamma-$ ), in both cases the Raman spectra obtained show bands corresponding to lycopene, thus demonstrating that lycopene has been encapsulated forming the complex with $\beta$ - and $\gamma$-CD.

The Raman spectrum of $\gamma$-CD/lycopene complex (Fig. 1c) shows a $v(\mathrm{C}=\mathrm{C})$ band which is slightly shifted towards higher wavenumbers $\left(1517 \mathrm{~cm}^{-1}\right)$. In the case of the $\beta$-CD/lycopene complex (Fig. 1e) the $v(\mathrm{C}=\mathrm{C}$ ) band undergoes a strong shift to $1528 \mathrm{~cm}^{-1}$, i.e. $16 \mathrm{~cm}^{-1}$ (Fig. 2). In addition a shoulder at $1546 \mathrm{~cm}^{-1}$ is also seen, 


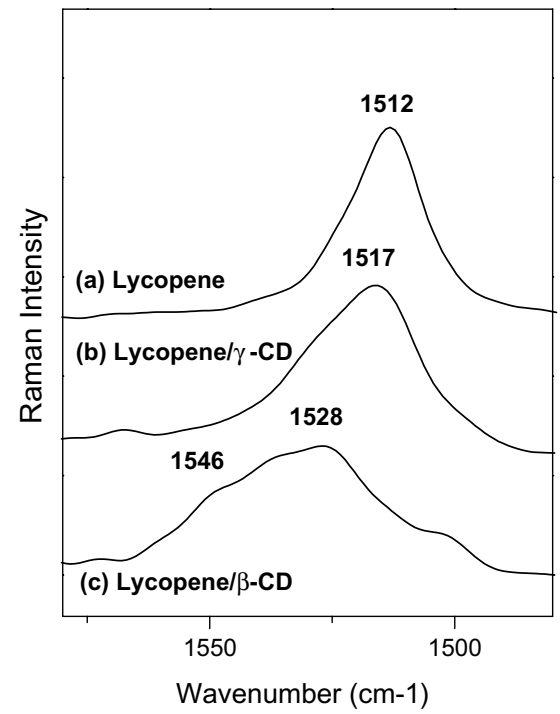

Fig. 2. Detailed micro-Raman spectra in the $\mathrm{C}=\mathrm{C}$ stretching region corresponding to all-trans-lycopene (a) and all-trans-lycopene complex with $\gamma$ - (b) and $\beta$ - cyclodextrin (c).

the strong shift upwards indicates that all-trans-lycopene undergoes a structural change upon complexation with $\beta$ $\mathrm{CD}$. On the other hand, the presence of several $\mathrm{C}=\mathrm{C}$ stretching bands might indicate that several lycopene structures are interacting with $\beta-\mathrm{CD}$. Besides, a broad band appears centred at $1004 \mathrm{~cm}^{-1}$ in the Raman of the $\beta-\mathrm{CD} /$ lycopene complex, which might be attributed to the $v(\mathrm{C}-$ C) vibrations in lycopene (Fig. 1e). The shifts observed for the $v(\mathrm{C}=\mathrm{C})$ and $v(\mathrm{C}-\mathrm{C})$ vibrations in opposite senses indicate that lycopene interacting with $\beta-\mathrm{CD}$ has a lower electronic conjugation degree in the polyene chain due to a conformational change induced by the complexation with the cyclodextrin molecule which is, in turn, induced by the strong intermolecular interaction. The interaction between cyclodextrins and lycopene takes place through hydrophobic bond due to the hydrophobic nature of lycopene and the inner cavity of cyclodextrins and in the case of the $\beta$ cyclodextrin complex, the structural changes are much stronger than in the $\gamma$-cyclodextrin complex, they are attributed to the better adaptation of the hydrophobic inner cavity of this host in relation to the ligand and also due to the smaller hydrophobic inner cavity in the case of the $\beta$-cyclodextrin, which seems to exert a higher structural modification on the lycopene structure.

On the other hand the lower Raman signal seen in the complex with $\beta-C D$ can be attributed to a lower resonance Raman effect of lycopene in the presence of this CD due to the stronger structural change induced in the dye, as described above.

The interaction with $\beta$-CD seems to also affect the carbohydrate structure. In fact the bands observed in the Raman spectrum at $867 \mathrm{~cm}^{-1}$ shift downwards to $860 \mathrm{~cm}^{-1}$, while that at $478 \mathrm{~cm}^{-1}$ is shifted upwards to $482 \mathrm{~cm}^{-1}$, thus indicating that a structural change involving the molecular skeleton and the $\mathrm{CH}_{2}$ groups is taking place. In contrast, the corresponding bands of $\mathrm{CD}$ in the $\gamma$-CD/lycopene complex (Fig. 1c) practically remained unchanged.

The changes observed in the present work for lycopene $v(\mathrm{C}=\mathrm{C})$ and $v(\mathrm{C}-\mathrm{C}) / \delta(\mathrm{C}-\mathrm{H})$ vibrations in the Raman spectrum have also been seen in the Raman of the $\beta$-carotene 15-cis isomer by Koyama, Takatsuka, Nakata, \& Tasumi, 1988. According to this, we suggest that all-trans- lycopene may undergo an isomerization into the cis-isomer during its encapsulation with $\beta$-CD. Specifically, the strong shift observed for these bands in the complex with $\beta$-CD points out that the double bond bearing the cis isomerization in bounded lycopene must be rather placed in a central position, leading to the $\beta-\mathrm{CD} /$ lycopene complex as also deduced from RMN (Yannakopoulou, Ripmeester, \& Mavridis, 2002). Since the length of lycopene is ca. $19 \AA$ and that of $\beta-C D$ is ca. $9 \AA$ we can also assume a double encapsulation of lycopene with two $\mathrm{CD}$ rings, in such a way that a cis-structure is induced in the middle of the dye chain. Similar channel encapsulation was found by

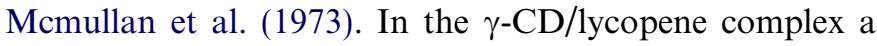
channel encapsulation is also possible, although in this case the all-trans structure seems to be preserved. As commented in Introduction, the cis isomer of lycopene is thermally less stable than the trans form, the isomerization of all-trans-lycopene during its encapsulation into CDs would appear, in principle, unimportant as long as the complex formed stabilizes the lycopene molecule. In addition, from Raman results the $\beta$-CD host seemed to be the most advantageous $\mathrm{CD}$ to encapsulation all-trans-lycopene using conventional methods as it preserves better the cis isomeric form existing in solution, which is the most active or the best form under the biological point of view to be adsorbed by the organism.

As far as the formation of the complex by using the supercritical fluid procedure is concerned we proved the stability of the pure cyclodextrins at different pressures. In spite of the growing processes released on the crystal structures of $\alpha$-, $\beta$ - and $\gamma$-cyclodextrins slight changes were observed in their crystalline state when increasing strength is applied from 85 to 245 bar, as shown in Fig. 3. This observation allows to assure the structural validity of the cyclodextrins in the pressure range considered.

Concerning, the SF- $\mathrm{CO}_{2}$ complexes, they were also, as mentioned in 2, studied by micro-Raman spectroscopy. As a result, the recovery of lycopene complex obtained in the first separation vessel was $67.5 \%$. Although the most complex percentage was collected in the first separation vessel, the second vessel was also considered to evaluate the amount of complex formed with the rest of lycopene. Fig. 4 presents the Raman spectra of the $\beta$-CD/lycopene complexes obtained in the first and second separation vessels (Fig. 4c and d, respectively). The Raman spectra obtained show bands at 1532, 1151 and $1007 \mathrm{~cm}^{-1}$ corresponding to lycopene (Fig. 4b), thus demonstrating that lycopene has been complexed forming the complex with $\beta-\mathrm{CD}$. Also the bands of $\beta-\mathrm{CD}$ are present (Fig. 4a). As 


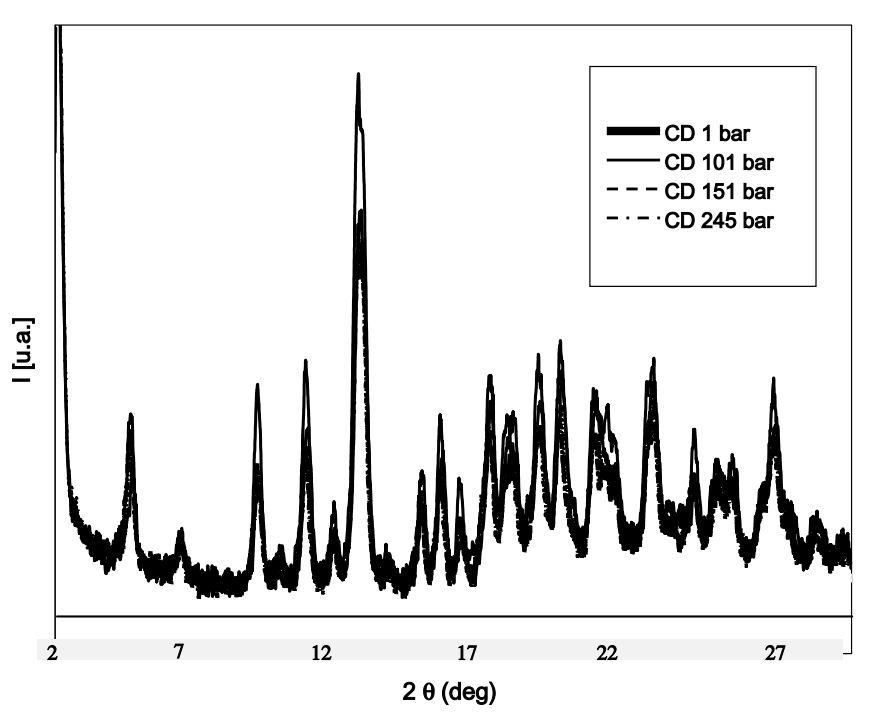

Fig. 3. Powder $\mathrm{X}$-ray diffraction patterns, of pure $\beta$-cyclodextin, obtained under different $\mathrm{SF}-\mathrm{CO}_{2}$ pressures $(1,101,151$ and $245 \mathrm{bar})$. The patterns were all registered at $50{ }^{\circ} \mathrm{C}$ except the one at 1 bar which was registered at room temperature.

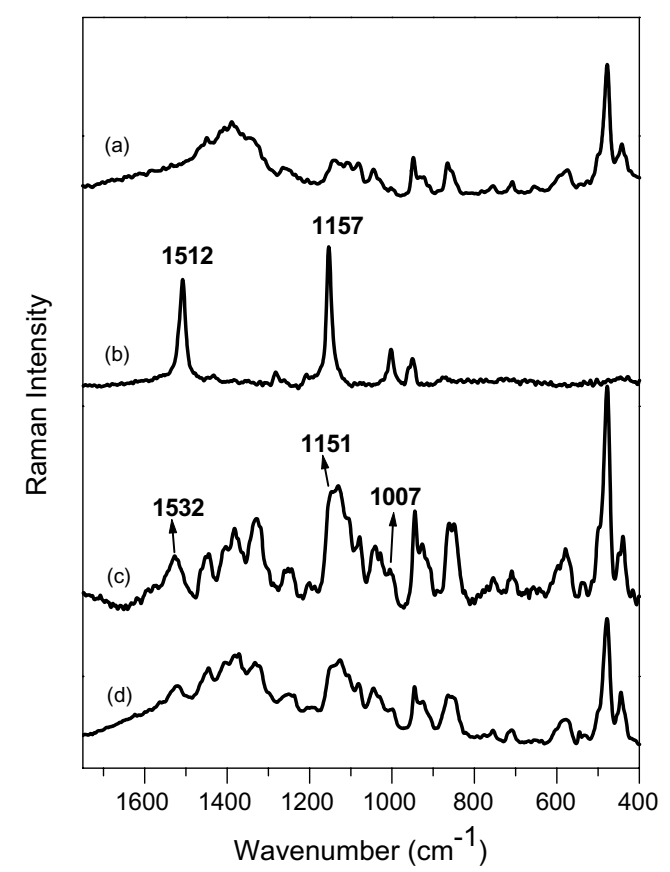

Fig. 4. Micro-Raman spectra representing the band intensity obtained at different wave number $\left(\mathrm{cm}^{-1}\right)$ corresponding to $\beta$-cyclodextrin (a), alltrans-lycopene (b) and all-trans-lycopene encapsulated with $\beta$-cyclodextrin by SF- $-\mathrm{CO}_{2}$ in the first separation vessel (c) and in the second separation vessel (d).

can be seen, the Raman signal of lycopene in these complexes, relative to that of the CD host, was weaker than those obtained in $\beta$-CD/lycopene complex encapsulated by conventional methods (Fig. 1e), thus a lower effectiveness of this method (SF) could be concluded from this results. The comparative analysis of the lycopene Raman bands indicates that there must be a conformational change in the ligand similar to that observed in conven- tional method. In fact, the $v(\mathrm{C}=\mathrm{C})$ band undergoes a large shift towards higher wave numbers (from $1512 \mathrm{~cm}^{-1}$ in the solid to $1532 \mathrm{~cm}^{-1}$ in the presence of $\left.\beta-\mathrm{CD}\right)$, while the $v(\mathrm{C}-$ $\mathrm{C}) / \delta(\mathrm{C}-\mathrm{H})$ band is shifted to lower wave numbers. This was again attributed to a conformational change from all-trans to cis-conformation.

By comparison of the two encapsulation procedures used in the present work, we could conclude that the conventional method appeared to provide better results in terms of encapsulation yield $(93.8 \%$ vs. $67.5 \%)$. However, it is important to keep in mind that the employment of SF- $\mathrm{CO}_{2}$ is an interesting alternative as it offers some important advantages. Among them, it could be highlighted the fact that the extraction, fractionation and encapsulation is accomplished in just one step, which shortens substantially the overall procedure $(3 \mathrm{~h}$ vs. $20 \mathrm{~h}$ corresponding to the conventional method). Additionally, the sample manipulation is far lesser and, thus the reliability of the obtained results is increased. Finally, the use of supercritical fluids in foods is highly recommended as it avoids the employment of large amounts of organic solvents.

As a final point, the stability study of the encapsulated obtained, as specified in 2 , reflected that no variation of the spectral signals shown by the $\beta-\mathrm{CD} / \mathrm{lycopene}$ complex was found even after 6 months. Consequently, we might conclude that lycopene complex with $\beta$-CD remains stable at least during half a year. This aspect allows to confirm the interest of the study performed in the present work.

\section{Acknowledgements}

This work was financially supported by CICYT (Project AGL2002-03615), by Dirección General de Investigación, Ministerio de Educación y Ciencia (Project FIS200400108) and by Comunidad Autonoma de Madrid (Project number GR/MAT/0439/2004). The authors thank Olga López Pérez for her help in performing some of the experimental work.

\section{References}

\section{AOAC (1984). Section 43.015.}

Basu, H. N., \& Del Vecchio, A. (2001). Encapsulated carotenoid preparations from high-carotenoid canola oil and cyclodextrins and their stability. Journal of American Oil Chemist Society, 78, $375-380$.

Chandler, L. A., \& Schwartz, S. J. (1987). HPLC separation of cis-trans carotene isomers in fresh and processed fruits and vegetables. Journal of Food Science, 52, 669-672.

Di Mascio, P., Katser, S., \& Sies, H. (1989). Lycopene as the most efficient biological carotenoid singlet oxygen quencher. Archives of Biochemistry Biophysics, 274, 532-538.

Garbow, J. R., Likos, J. J., \& Schroeder, S. A. (2001). Structure, dynamics, and stability of $\beta$-cyclodextrin inclusion complexes of asparme and neotame. Journal of Agricultural and Food Chemistry, 48, 2053-2060.

Gómez-Prieto, S., Ruiz del Castillo, M. L., Santa-María, G., Blanch, G. P., \& Herraiz, M. (2002). Extracción fraccionada de carotenoides mediante fluidos supercríticos. Patent n. WO 03/103645A1, CSIC. 
Koyama, Y. (1995). Resonance Raman spectroscopy. In G. Britton, S. Liaaen-Jenson, \& H. Pfander (Eds.). Carotenoids (Vol. 1B, pp. 135-146). Basel, Switzerland: Birkhaeuser.

Koyama, Y., Takatsuka, I., Nakata, M., \& Tasumi, M. (1988). Raman and Infrared Spectra of the All-trans, 7-cis, 9-cis, 13-cis and 15-cis isomers of $\beta$-carotene: Key bands distinguishing stretched or terminalbent configurations from central-bent configurations. Journal of Raman Spectroscopy, 19, 37-49.

Leyton, P., Sanchez-Cortes, S., García-Ramos, J. V., Domingo, C., Campos-Vallette, M. M., Saitz, C., et al. (2004). Selective molecular recognition of polycyclic aromatic hydrocarbons (PAHs) on calix[4]arene-functionalized $\mathrm{Ag}$ nanoparticles by surface-enhanced Raman scattering. Journal of Physical Chemistry B, 108, 17484-17490.

Li, S., \& Purdy, W. C. (1992). Cyclodextrins and their applications in analytical chemistry. Chemistry Review, 92, 1457-1470.

Matioli, G., \& Rodriguez-Amaya, D. B. (2003). Microencapsulation of lycopene with cyclodextrins. Ciênc. Tecnol. Aliment. Vol. 23 (pp. 102-105). online at: $<$ http://www.scielo.br/scielo.php?script=sci_arttext\&pid $=$ S0101-20612003000400019\&lng $=$ en\&nrm $=$ iso $>$.

Mcmullan, R. K., Saenger, W., Fayos, J., \& Mootz, D. (1973). Topography of cyclodextrin inclusion complexes. Carbohydrate Research, 31, 37-46.

Mele, A., Mendichi, R., \& Selva, A. (1998). Non-covalent associations of cyclomaltooligosaccharides (cyclodextrins) with trans- $\beta$-carotene in water: evidence for the formation of large aggregates by light scattering and NMR spectroscopy. Carbohydrate Research, 310, 261-267.

Mele, A., Mendichi, R., Selva, A., Molnar, P., \& Toth, G. (2002). Noncovalent associations of cyclomaltooligosaccharides (cyclodextrins) with carotenoids in water. A study on the $\alpha$ - and $\beta$-cyclodextrin $/ \varphi, \varphi$ carotene (lycopene) systems by light scattering, ionspray ionization and tandem mass spectrometry. Carbohydrate Research, 337, $1129-1136$.

Nguyen, M. L., \& Schwartz, S. J. (1999). Lycopene: chemical and biological properties. Food Technology, 53, 38-45.
Pedrolas Islas, R. (2002). Alimentos microencapsulados. Particularidades de procesos para la microencapsulación de alimentos para larvas de especies acuícolas. In: L. E. Cruz Suárez, D. Ricque-Marie, M. TapiaSalazar, M. G. Gaxiola-Cortés, \& N. Simies, (Eds). VI International Simposium of Aquatic Nutrition. México.

Perrut, M., Jung, J., \& Leboeuf, F. (2005). Enhancement of dissolution rate of poorly soluble active ingredients by supercritical processes. Part II: Preparation of composite particles. International Journal Pharmaceutics, 288, 11-15.

Rekharsky, M. V., \& Inove, Y. (1998). Complexation thermodynamics of cyclodextrins. Chemistry Review, 98, 1875-1917.

Shahida, F., \& Han, X. (1993). Encapsulation of food ingredients. Critical Reviews in Food Science and Nutrition., 33, 501-547.

Shi, J., \& Le Maguer, M. (2000). Lycopene in tomatoes: chemical and physical properties affected by food processing. Critical Reviews in Food Science and Nutrition, 40, 1-42.

Stahl, W., \& Sies, H. (1996). Lycopene: a biologically important carotenoid for human? Archives of Biochemistry and Biophysics, 336, $1-9$.

Szente, L., \& Szejtli, J. (2004). Cyclodextrin as food ingredients. Trends in Food Science \& Technology, 15, 137-142.

Tu, A. (1982). Raman spectroscopy in biology. New York: John Wiley \& Sons.

Wilberg, V. C., \& Rodriguez Amaya, D. B. (1995). HPLC quantitation of major carotenoids of fresh and processed guava, mango and papaya. Lebensmittel-Wissenschaft + Technologie, 28, 474 480.

Withnall, R., Chowdhry, B. Z., Silver, J., Edwards, H. G. M., \& de Oliveira, L. F. C. (2003). Raman spectra of carotenoids in natural products. Spectrochimica Acta A, 59, 2207-2212.

Yannakopoulou, K., Ripmeester, J. A., \& Mavridis, I. M. (2002). Controlled release of the Prays Oleae pheromone, as a consequence of supramolecular structure: study of the Z-7-tetradecenal $/ \beta$-cyclodextrin complex in the solid state and in solution. Journal of the Chemical Society-Perkin Transaction, 2, 1639-1644. 